\title{
The third culture
}

Is quantum physics, like science and literature, in a world of its own?

\section{Quantum: A Guide for the Perplexed \\ by Jim Al-Khalili \\ Weidenfeld \& Nicolson: 2003. 192 pp. 118.99 \\ Sterling Publications: 2003. \$24.95 \\ Frank Wilczek}

C. P. Snow famously wrote of "two cultures", scientific and literary, and of the difficulties of communication between them. To participate in the scientific culture one must be comfortable with the guiding principles of quantitative thought and strict logic, and a body of basic facts; to participate in the literary culture one must be familiar with a canon of works and expressive techniques. There are real difficulties in reconciling the two, of course, but with some effort and goodwill they can be whittled down. Scientists Freeman Dyson and Oliver Sacks, historian William McNeill and author Richard Powers are a few outstanding examples of people whose contemporary work has straddled these cultures successfully.

Problems of a different order arise when we come to "without doubt the single most important scientific advance of the twentieth century", as the author of Quantum (correctly, in my opinion) describes quantum theory. Coming to terms with quantum theory requires not just quantitative thought, but the systematic replacement of unreliable intuition, derived from everyday experience, by mathematical abstraction; not just strict logic, but expanded concepts of meaning and uncertainty; not just familiarity with some basic facts, but imaginative reconciliation of apparent contradictions. Because of these difficulties, the quantum revolution has not yet had the deep impact on literary and, ultimately, common culture that its power and novelty merit.

The relatively small band of illuminati who have been initiated into the quantum culture view the world in a new and richer way. They live in a world not of four or eleven dimensions, but of infinitely many. They learn to dance "on the border between genius and madness", enjoying "the highest form of musicality in the sphere of thought", as Einstein variously described it. They can come to understand why chemistry works, the physical basis of quasi-magical technologies (such as microelectronics, lasers, superconductivity and magnetic resonance imaging), and the dreams of nanotechnology and quantum computing. And only they can begin to appreciate the weird but compelling concepts and algorithms that Nature uses as fundamental building blocks.

Not everyone has the time or patience to become a licensed quantum mechanic, of course. But many more thinking people may like to be initiated into this third culture, to experience a real vision of its perspective. Certainly any aspiring philosopher (lover of insight) or theologian (interpreter of ultimate reality), or anyone who wants to imagine the technology and economy of the not-sodistant future, must become so initiated.

It is to this audience, and this purpose, that Jim Al-Khalili's new book is addressed. It is a laudable attempt to make basic concepts of quantum theory, and some of their most important applications, more widely accessible. I don't think it is the ultimate book on the subject, or even the best available, but it is a worthy effort and could serve its intended audience well.

Written in a colloquial style, the book has the feeling of a conversation with the author. You sense his sincerity and enthusiasm throughout. It makes, especially in the early chapters, for easy, charming reading. There are no equations, and many pretty pictures; if anything, too many and too pretty - quite a few of the illustrations carry little if any information content, and these may become distracting or even irritating.

There are ten chapters, of which the first six are the best. They provide a detailed account of the classic two-slit experiment and the difficulties it poses for a particle interpretation; a bit of the early history of quantum theory; and a sketch of the ongoing debate over its interpretation. All this is done in a careful and leisurely way, with sensible judgements about when to draw back from technicalities and what to leave out. I can easily imagine an intelligent but initially uninformed reader thinking through this material while reading it, having questions arise and get answered, and coming to genuine understanding.

Things begin to slip away in the seventh chapter, "The subatomic world", which contains an extremely compressed account of atomic and nuclear physics. By this point we have shifted gears, passing from the intense discussion of a few basic ideas to glances at many. Things go seriously awry in the eighth, "The search for the ultimate theory", which is a sketch of some advanced ideas and speculations. The author is unsure of his ground here, and falls into a few outright errors. Supersymmetry does not relate electroweak interactions to quantum chromodynamics (QCD), and the Planck length is not the size of a quantum of space.
A more subtle and forgiveable mistake, which I mention here because it is both widespread and instructive, is the claim that quantum electrodynamics (QED) provides a sufficient foundation for the physics of chemistry and biology. In reality we need to input the masses and properties of atomic nuclei if we are to account for molecular vibrations and rotations, and motion in general. These nuclear properties are governed by QCD, the theory of quarks and gluons, not by electrodynamics.

Also hyper-compressed is the ninth chapter, "Putting the quantum to work". It provides an account of the applications of quantum physics, including semiconductor electronics, magnetic imaging, superconductivity, Bose-Einstein condensates and more, all in 30 pages of liberally illustrated large print. To be sure, a rapid survey style works better for this more grounded, less intricately layered material.

The tenth chapter, "Into the new millennium", returns to the spirit and material of the earliest chapters. It is a tantalizing introduction to some speculative possibilities for exploiting basic quantum phenomena (such

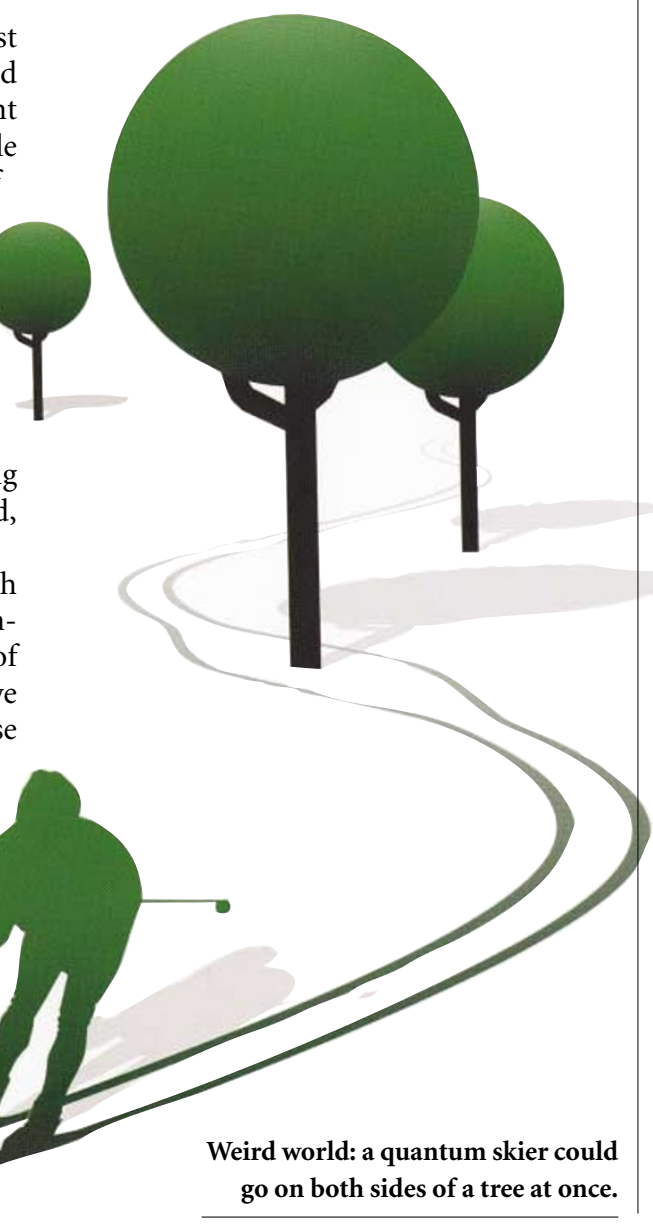


as entanglement and complementarity) in new forms of information processing and communication. I wish the author had gone deeper in pursuit of these logical outgrowths of his core themes, instead of scampering to touch all bases.

For seekers of the third culture, I'd still recommend Richard Feynman as the best guide. Try The Character of Physical Law (MIT Press, 1967), the early chapters in each volume of The Feynman Lectures on Physics and, for the ambitious, QED: The Strange Theory of Light and Matter (Princeton University Press, 1986).

Frank Wilczek is at the Center for Theoretical Physics, Massachusetts Institute of Technology, 77 Massachusetts Avenue 6-305, Cambridge, Massachusetts 02139-4307, USA.

\section{Taming the world?}

\section{Our Own Devices: The Past and}

Future of Body Technology

by Edward Tenner

Knopf: 2003. 336 pp. \$26.

\section{Howard P. Segal}

The unintended effects of technological developments may be more harmful than beneficial. This was the message - familiar to scholars but not to the general public that Edward Tenner discussed in original and amusing ways in his previous book Why Things Bite Back: Technology and the Revenge Effect (see my review in Nature 382, 504-505; 1996). In his latest book, Our Own Devices, Tenner examines the ceaseless interplay between technological devices and technique, or our ability to use them.

Most students of technology, he observes, concentrate on the devices themselves; their applications are usually harder to analyse. Yet Tenner also rejects the influential arguments of social critic Jacques Ellul that technique, broadly defined, has steadily shaped modern "technological society" and that its influence is inescapable. (The original French title of Ellul's most influential book, The Technological Society, was La Technique.)

Tenner focuses on several "body technologies" used in everyday life, and moves literally from toe to head: sandals, training shoes, chairs for office and home, musical key-

boards, text keyboards, babies' bottles,

glasses and helmets. Overall, these technologies have provided us with a more comfortable, efficient and protected existence as we have modified the natural world - which is how the earliest technologies began. Invariably, though, there are those "revenge effects". But Our Own Devices goes further than the first book in demonstrating how far body technologies have defined us as human beings.

Babies' bottles are usually the first technology that most of us encounter, but the technique of nursing must still be mastered by most children and mothers who seek to utilize both bottles and breasts. Regular use of rubber bottle nipples often diminishes infants' use of jaws and tongues to breastfeed. And too much baby formula may increase the prospects for certain adult ailments.

To take another example, most of us remain indebted to Roman sandals for the basic model of walking comfort in warmer climates. But comfort is not always a necessity, as the world's billion barefoot inhabitants could attest. For the rest of us, though, sandals - and shoes - make our feet too sensitive to manage without them. Yet cultural differences may dictate our particular choices, so Japanese men wearing geta (raised clogs) walk differently from their Western counterparts.

The role of literacy is critical to Tenner's analysis of glasses, chairs and keyboards, all of which affect us both physically and intellectually. Not surprisingly, glasses go hand-in-hand with mass literacy — but so does myopia. Ironically, glasses may cause children's eyes to grow differently, and so increase myopia as much as correct it. Meanwhile, a few straight-backed chairs for monks and clerks existed before ordinary people learned to read and write, but only with mass literacy did such chairs — and later reclining chairs - become fundamental to those enterprises. Reclining chairs, however, often weaken our back muscles, causing us to recline still more to be comfortable.

Initially, keyboards were used to spread musical literacy but later became critical for putting words on paper with typewriters. Yet many children's writing skills fail to develop properly because they spend ever more time on the computer keyboards that even-

tually followed.
Helmets provide one of Tenner's most intriguing examples. They were invented in classical times, but their success prompted the development of more powerful weapons and, Tenner claims, started the arms race. Adapted for sports, stronger, and presumably safer, helmets have ironically led to ever more violent contact and greater injuries.

However old in origin, all of Tenner's examples in their current forms reflect the sea change in conceptions about technology, in the West at least, over recent decades. We seem ever more concerned with technology providing small-scale fulfilment of individual needs and desires than with providing large-scale solutions to widely acknowledged social problems - from insufficient energy, communications and transportation systems to excessive poverty and population. Body technologies, rather than space programmes or nuclear power plants, have become our primary association with technology and technique. Tenner is not concerned with this trend, and seems happy to instruct and entertain in a non-ideological, often wry manner.

Tenner then briefly discusses the proliferation of mobile phones and palm pilots. Despite the dominance of the index finger, which represents 'authority', Tenner predicts the rise of the thumb: "when extended in the almost lost art of hitchhiking, the thumb shows the right attitude toward the future,
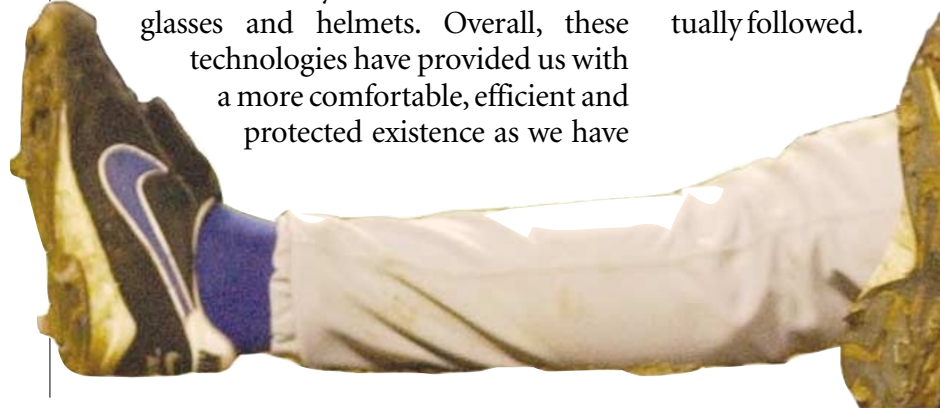